\title{
RISCAPE: an analysis of the international landscape of research infrastructures in astronomy and astroparticle physics.
}

\author{
Rob L.J. van der Meer ${ }^{1}$ \\ ASTRON (Netherlands Institute for Radio Astronomy) \\ Dwingeloo, the Netherlands \\ E-mail: meereastron.nI

\section{Carla Baldovin, Marjan Timmer, René Vermeulen} \\ ASTRON (Netherlands Institute for Radio Astronomy) \\ Dwingeloo, the Netherlands \\ E-mail: baldovineastron.nl, timmereastron.nl, rvermeulendastron.nI
}

The H2020 project RISCAPE studies the international research infrastructure landscape. The project will provide a systematic, focused, high-quality, comprehensive, consistent and peerreviewed international landscape analysis report on the position and complementarities of the major European Research Infrastructures (RIs) in the international research infrastructure landscape. ASTRON will collect the information on astronomy and astroparticle physics. As coordinator of the H2020 RI cluster project ASTERICS, ASTRON has a good network in European and worldwide astronomy and astroparticle physics research infrastructures. The final report with the landscape analysis will be available at the end of 2019. The ASTERICS community is a stakeholder in the RISCAPE project. With this presentation and publication we bring the RISCAPE endeavour and results to the attention of the ASTERICS community.

The New Era of Multi-Messenger Astrophysics - Asterics 2019

25 - 29 March, 2019

Groningen, The Netherlands 


\section{Introduction}

The goal for the RISCAPE [1] (European Research Infrastructures in the International Landscape) project is to provide a systematic, focused, high-quality, comprehensive, consistent and peer-reviewed international landscape analysis report on the position and complementarities of the major European Research Infrastructures (RIs) in the international research infrastructure landscape. The project covers eight disciplines:

- Environmental sciences

- Digital humanities, language studies and cultural heritage

- Social sciences

- Biomedical sciences

- Astronomy and astroparticle physics

- Physics and engineering

- e-infrastructures

- Energy

\subsection{ASTRON contribution to RISCAPE}

ASTRON (Netherlands Institute for Radio Astronomy) leads the work package in astronomy and astroparticle physics. ASTRON coordinates the ASTERICS RI cluster, and was therefore already at the beginning of the project well positioned to lead the astronomy and astroparticle physics domain search. The ASTERICS cluster includes all ground-based astronomy and astroparticle physics RI landmarks and projects in the ESFRI Roadmap 2016 [2]. These are the Extremely Large Telescope (ELT), Square Kilometer Array (SKA), Cherenkov Telescope Array (CTA), Cubic Kilometer Neutrino Telescope (KM3NeT) and European Solar Telescope (EST), covering the new major facilities with European contribution. ASTERICS furthermore engages with existing infrastructures and projects like the European Gravitational Observatory (EGO), the Joint Institute for VLBI ERIC (JIV-ERIC) and the European Virtual Observatory (EURO-VO). During the RISCAPE project, we used the existing ASTERICS network in Europe and our other connections to extend to worldwide coverage.

\subsection{Limiting the scope}

The research in the astronomy and astroparticle physics domain takes place through both ground based and space based facilities. The space based facilities in the European context are almost all coordinated or organised through the European Space Agency, ESA. The large international space based facilities are coordinated by a handful of national agencies. We therefore consider the lanscape of space based research as known and will exclude it from our studies; only ground based facilities will be studied. 


\section{RISCAPE International Landscape analysis concept}

A landscape analysis is a type of organizational analysis, where information of the main actors of some specified field are systematically collected. The landscape analysis in this context refers to a process where the European RIs are evaluated on their complementarity to key international (i.e. outside of Europe) RIs and initiatives. This process consists of specific steps and is aimed towards producing a report that is fair and representative of the global landscape. The project will include the following phases:

1. Select and engage the European RIs for the analysis (European Engagement).

2. Analyse the complementarity of European RIs in respect to the international RI landscape (International engagement).

3. Integration, consistency, evaluation and dissemination.

\subsection{Complementarity}

A key factor in the analysis step is the concept of complementarity, which can be evaluated from the axes that are relevant to the discipline and the RIs to be analysed. The three axes used here are Technical, Geographical and Challenge complementarities (see Figure 1) and are defined as follows:

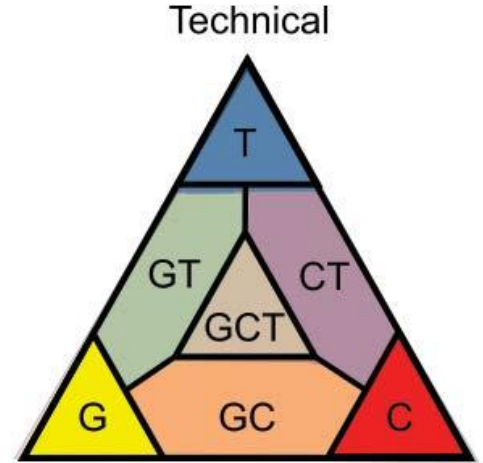

Geographical Challenge

Figure 1: Complementarity axes in multidisciplinary Research Infrastructure landscape

Technical (T) complementarity means that the European RI complements the technical capabilities of the international RI field - this can be e.g. differences in observation capabilities (detection energies, spatial or temporal resolution, and so on). In the current ESFRI landscape document, the physics and engineering landscape was presented mostly from the Technical complementary point of view. Purely Technical RI complementarity describes a situation where the European and the international RI only differ by technical capabilities, and the other aspects are either similar or insignificant. (astronomy example: ELT, LSST, SKA.)

Geographical (G) complementarity means that the European RI complements international RIs and initiatives by providing services or data about a specific geographical area, often Europe. These RIs are typical in the environmental sector. (astronomy example: Virgo - LIGO, CTA northsouth.)

Challenge (C) complementarity presents a difference in the overall scientific aims of the RI, which can reflect the operational goals and products developed in the RI, even if the other factors would be similar. High level of complementarity in Challenges can represent a sitution where otherwise similar RIs answer different social or technical questions - also showing high potential 
of e.g. data sharing or widening the user base for other challenges. (Astronomy example: ORCA and ARCA sites of KM3NeT.)

\subsection{Challenges in the RI landscape analysis in RISCAPE}

In order to create consistent, high-quality reports on the analysis in each work package, RISCAPE organized a special work package for comparing and aligning the way the teams in the various areas started working. Together we defined the common way all teams should work in collecting and analyzing their information. We determined three major challenges where work needed to be aligned. These are described in the following paragraphs.

\subsubsection{Terminology}

We needed to determine what terminology to use and find out if we all speak the same language, between the various work packages and science domains and between the various regions in the world.

The term "Research Infrastructure" is not uniformly and globally defined. However, for the purpose of RISCAPE, the following requirements are defined.

The research infrastructure:

- has science or research in its core activities,

- is longstanding and its time horizon is longer than a research project,

- offers services to users outside the infrastructure,

- reaches scientific impact that is expected from ESFRI landmarks or projects.

\subsubsection{Finding the RIs}

In order to find the RIs in Europe and worldwide RISCAPE engaged with the ESFRI RIs via the cluster projects (like ASTERICS for astronomy and astroparticle physics). The European RIs and smaller projects were requested to provide the names of similar initiatives at international level that have minimum or non-existing contribution from EU countries. The list was complemented with an extensive research of the publicly available national and regional science roadmaps.

Another important question was, how to determine the completeness and usefulness of the list. RISCAPE established a stakeholder panel with high-level policymakers from the various domains. We expect that together with them we cover enough knowledge to determine the gaps in the list. The initial analysis in some fields showed that the direct top-down approach, using contacts at ministries, funding agencies, etc. did not find all possible RIs. We noticed that some areas in the world did not show many potential RIs. Therefore, we asked the scientists active in the research field. They follow the literature and can have direct links to other specialist, inside and just outside their main field. 


\subsubsection{Getting the right information}

RISCAPE wants to collect information on as many RIs as possible and on each of them as complete as possible. Collecting detailed information from many sources is often done by distributing a questionnaire. We were afraid that just using a questionnaire would not work. People receiving the questionnaire might no be the optimal persons in their organisation, they might forget or fail to complete the questionnaire and the answers might not align with the questions. The RISCAPE approach was to minmize the effort needed from the contact person at the RI:

- We searched for initial contact points preferably from warm contacts from EU RIs or scientists. A personal contact is often needed and increases the chances of success.

- We pre-filled the online questionnaire with information retrieved from the web.

- We asked the contact person to correct and complete the online form.

- We performed structured interviews by telephone around the questions in the online form to prevent and remove misunderstandings. We took time for friendly conversation to get to know each other and create a genuin two-way communication. For optimal result, the interviewer should have domain expertise.

- We sent the final set of collected data for confirmation. This builds trust.

- We used a common database over all work packages/domains for traceability of data.

\section{The process in the astronomy and astroparticle physics domain}

Figure 2: RISCAPE project timeline in spring 2019

\begin{tabular}{|c|c|c|c|c|}
\hline Preparation phase & Research phase & Analysis & Deepening analysis & Results \\
\hline $\begin{array}{l}\text { Defining the } \\
\text { methodology. }\end{array}$ & $\begin{array}{l}\text { Collecting } \\
\text { information from } \\
\text { international Rls. }\end{array}$ & $\begin{array}{l}\text { Finalising information } \\
\text { collection. }\end{array}$ & $\begin{array}{l}\text { Combining landscape } \\
\text { analyses for final } \\
\text { analysis. }\end{array}$ & $\begin{array}{l}\text { Publication of final } \\
\text { report. }\end{array}$ \\
\hline $\begin{array}{l}\text { Setting up the } \\
\text { stakeholder panel. }\end{array}$ & $\begin{array}{l}\text { Consolidation and } \\
\text { quality control. }\end{array}$ & $\begin{array}{l}\text { Workshop with } \\
\text { stakeholders. }\end{array}$ & $\begin{array}{l}\text { Preparing the final } \\
\text { report }\end{array}$ & \\
\hline $\begin{array}{l}\text { Engagement with } \\
\text { European RIs and } \\
\text { clusters. }\end{array}$ & & $\begin{array}{l}\text { Analysing different } \\
\text { domain landscapes. }\end{array}$ & $\begin{array}{l}\text { Disseminating the } \\
\text { results. }\end{array}$ & \\
\hline
\end{tabular}

Following the description in section 2, we started the process from the RIs in the ESFRI Roadmap and collected several warm and new contacts in International RIs. The initial plan was very ambitious. It turned out that more effort than expected went into mobilizing people both in the European and in the international RIs to give us the information we needed. That resulted in a shift of the planning. The planning at the spring of 2019 is shown in Figure 2. 


\section{The outcome}

The European research infrastructures provided us with the names of their international counterparts (meaning non-European) and contact names. The list of international research infrastructures was later verified and complemented by studying national roadmaps and other policy documents. We finally produced an initial list of 55 international RIs in Astronomy and Astroparticle physics that were invited to take part in the study. With only a few of these, an active and enthusiastic exchange of information was easily established. With others we are still at the first stage of establishing contact and setting up an appointment for the survey and interview.

Over the course of the RISCAPE project, some RIs that did not provide services to scientists outside their collaboration network, are now changing their ideas and starting to allow archival research on their data, or even giving access to recent observations. All of this due to the multimessenger astronomy activities that have become more common since the first gravitational wave observations. Movements like this show that the landscape analysis will only be a snapshot in time. Information from the landscape analysis should be used with caution for completeness and updated regularly. Care should be taken not to exclude RIs in the future based on our current comparison.

\section{Conclusion}

RISCAPE will describe the level and type of complementarity between European and international RIs, identifying major gaps and opportunities for profitable linking or collaborations. The final public report will directly benefit the European Union's strategic RI development and policy.

\section{Acknowledgements}

The RISCAPE project is funded by the European Union (EU) Horizon 2020 program under Grant number 730974.

\section{References}

[1] https://riscape.eu/

[2] https://www.esfri.eu/sites/default/files/20160308_ROADMAP_single_page_LIGHT.pdf 